\title{
Preoperative management of hypoplastic left heart syndrome
}

\author{
SANDRO DESSARDO $(\bowtie) \bullet$ \\ VLADIMIR AHEL \\ Pediatric Department, University Hospital \\ Rijeka \\ Istarska 43, HR-51000 Rijeka, Croatia \\ Phone: +38598459225 \\ E-mail: sandro.dessardo@medri.hr
}

\section{SANDRO DESSARDO • VLADIMIR AHEL}

\begin{abstract}
Pediatricians are frequently involved in the care of cyanotic newborns in the labor and delivery room, as well as in the well baby nursery. Causes of hypoxia and cyanosis in the term newborn can be found within all physiological systems. Congenital heart structural diseases account for the largest diagnostic category. There have been significant advances during the past years in the diagnosis and treatment of neonates with critical congenital heart disease, especially in the field of pre- and post-operative intensive care.

The term hypoplastic left heart syndrome (HLHS) describes a spectrum of cardiac structural abnormalities characterized by marked hypoplasia of the left ventricle and ascending aorta. Prenatal diagnosis, initial resuscitation and optimal preoperative management are key elements that allow the best opportunity for low mortality and normal neurodevelopment in affected newborns. Preoperatively, the goal is to achieve adequate systemic oxygen delivery. Patency of the ductus arteriosus (DA) is critical for survival until surgery. Blood flow to the pulmonary and systemic circulations should be nearly balanced (goal $Q_{p} / Q_{s}$ ratio of 1). The immediate therapy for all infants with $H L H S$ is an intravenous infusion of prostaglandin $E_{1}\left(P G E_{1}\right)$ in order to manipulate the DA and maintain ductal patency. Oxygen saturations of $75 \%$ to $85 \%$ by pulse oximetry suggest an adequate balance between systemic and pulmonary blood flow. Judicious use of inotropic support is initiated if evidence of low cardiac output is detected. Diuretics may be necessary to help alleviate the increased volume load on the right ventricle. The goal of respiratory management is to increase pulmonary vascular resistance and decrease systemic vascular resistance. Infants with HLHS who are born with a severely restricted or no inter-atrial communication, a rare occurrence, have profound hypoxemia. The severe restriction of blood flow across the atrial septum results in a life-threatening situation and these patients, which present with severe cyanosis and hemodynamic instability, require urgent postnatal cardiac catheterization to relieve the septal obstruction and improve oxygenation. Special attention should be paid to the prevention of brain injury and poor neurodevelopmental outcome.

Care for infants with HLHS is complex, and often multiple specialists are involved. Despite an increase in the number of newborns with complex congenital heart disease and a growing percentage of patients with single-ventricle physiology, it is possible to care for this particular group of patients and achieve acceptable mortality risks, even in centres with no pediatric cardiac surgery facilities, if good preoperative management protocols are followed.
\end{abstract}

Key words: congenital heart disease, newborn, intensive care, hemodynamics, PGE1, ductus arteriosus

\section{Introduction}

O tempora, o mores. Too often, little interest is shown toward the intensely cyanotic and desperately ill newborn because the majority of causative lesions do not lend themselves to correction and infrequently to satisfactory palliation, stated Hall and co- workers in Circulation in 1965. (1) At that time the reported survival rates for newborns with structural heart disease ranged from $31 \%$ to $37 \%$. It was only 15 years later that Izukava et al. reported the increasing survival rate in newborns with CHD (Congenital Heart Disease) at $70 \%$, in the first month after surgery. (2)

Pediatric care providers are repeatedly called upon to evaluate a cyanotic newborn in the labor and delivery room, as well as in the well baby nursery. Causes of hypoxia and cyanosis in the term newborn can be found within all physiological systems and take the form of hundreds of specific diagnoses. Among them, congenital heart structural diseases account for the largest diagnostic category.

There have been significant advances during the past years in the diagnosis and treatment of neonates with critical congenital heart disease, especially 
in the field of pre- and post-operative intensive care. (3)

Optimal preoperative care involves / $1 /$ primary stabilization, airway management and establishment of vascular access; /2/ complete noninvasive definition of structural defects; /3/ evaluation and treatment of secondary organ dysfunction; /4/ cardiac catheterization.

Congenital heart diseases are complex and can be difficult to categorize or conceptualize. Rather than trying to determine the management for each individual anatomical defect, a physiological approach can be taken. The basic issues that should be evaluated are /1/ the way in which the systemic venous return reaches the systemic arterial circulation; /2/ the characteristics of the connection between the pulmonary and systemic circulation; /3/ whether the defects are amenable to a two-ventricle or single-ventricle repair; /4/whether pulmonary blood flow is increased or decreased; /5/ whether there is a volume load or pressure load on the ventricles;

Hypoplastic left heart syndrome (HLHS) is the ultimate challenge for the neonatal and/or pediatric intensivist. Despite the advances in the management of HLHS, due to the development of new surgical techniques, it remains the most retractable congenital heart disease with a high mortality rate. However, by carrying out appropriate preoperative management the preoperative condition can be stabilized in most cases, even in patients with heart failure on admission. (4)

The term HLHS describes a spectrum of cardiac structural abnormalities characterized by marked hypoplasia of the left ventricle and ascending aorta. The aortic and mitral valves may be atretic, hypoplastic or stenotic. Unless a large patent ductus arteriosus (DA) supplies the systemic circulation, the syndrome is uniformly lethal in the immediate postpartal period. Prenatal diagnosis, initial resuscitation and optimal preoperative management are key elements that allow the best opportunity for low mortality and normal neurode- velopment in affected newborns. (5) Peri-operative mortality is a significant concern, and patients with HLHS are at risk preoperatively, intraoperatively, and especially postoperatively.

\section{Preoperative management}

A fundamental understanding of the role of pulmonary and systemic resistance in determining blood flow and adequate interpretation of systemic arterial and venous oxygen saturations $\left(\mathrm{SaO}_{2}\right.$ and $\mathrm{SVO}_{2}$, respectively) are crucial to the optimal clinical management of infants with HLHS from the very beginning. (6) The neonate with HLHS has a very complex cardiovascular physiology. The oxygenated blood returning through the pulmonary veins to the left atrium cannot flow into the left ventricle because of the dysfunctional mitral valve. Accordingly, it crosses the atrial septum through a septal defect and mix with the desaturated blood in the right atrium. The mixed blood leaving the right ventricle flows to the lungs via the pulmonary arteries and to the body via the open DA. Hence, fully open, unrestrictive, communications through the atrial septum and DA are the main conditions of survival for a newborn with HLHS.

Clinically, the syndrome typically presents within the first 24 to 48 hours of life, when the constriction of the DA causes a decrease in systemic blood flow, shock and eventually death. Infants with an absent or restrictive interatrial communication may present earlier.

\section{Open the ductus}

Preoperatively the goal is to achieve adequate systemic oxygen delivery. Patency of the ductus arteriosus is critical for survival until surgery. Blood flow to the pulmonary and systemic circulations should be nearly balanced (goal $Q$ $\mathrm{p} / \dot{Q}_{\mathrm{S}}$ ratio of 1$)$. The immediate therapy for all infants with HLHS is an intravenous infusion of prostaglandin $E_{1}$ $\left(\mathrm{PGE}_{1}\right)$ in order to pharmacologically manipulate the DA and maintain ductal patency. A continuous infusion of the prostaglandin is initiated, preferably through a central catheter, at a rate of
0.05 to $0.1 \mu \mathrm{g} / \mathrm{kg}$ per minute. However, numerous side effects are associated with $\mathrm{PGE}_{1}$ infusion such as respiratory depression, fever, lethargy, irritability, myoclonic jerks, flushing, edema, pyloric stenosis, hyperostosis, necrotizing enetrocolitis, as well as structural remodeling of the DA and the pulmonary vessels, with a reported incidence of these complications ranging from 10 to $40 \%$. $(7,8)$ In the future Sildenafil could be a reasonable alternative to $P G E_{1}$ for maintaining DA patency, since it can also prevent and reverse DA closure through a mechanism that is distinct, and eventually safer, from the PGE 1 mechanism. (9) An audible murmur and adequate peripheral perfusion provide evidence of ductal patency; however, Doppler echocardiography is needed to confirm flow. Once the ductus is open, the rate of infusion may be reduced to decrease the risk for potential adverse effects. Unrestricted blood flow through the ductus arteriosus is necessary for systemic perfusion. Sometimes even a temporary discontinuation of the prostaglandin infusion is possible, with careful monitoring of blood pressure and urine volume as well as frequent echocardiographic examinations, in order to enable maintenance of balance between systemic and pulmonary blood flow. (4)

\section{Balance the circulation}

The $\dot{Q}_{\mathrm{p}} / \dot{Q}_{\mathrm{S}}$ ratio preoperatively is dictated by the adequacy of the interatrial communication. An infant with a mildly restrictive interatrial communication may have balanced circulation and remain in a clinically stable condition as long as the ductus arteriosus remains open. Oxygen saturations of $75 \%$ to $85 \%$ by pulse oximetry suggest adequate balance between systemic and pulmonary blood flow. Ventilatory support may be needed for apneic episodes or tenacious secretions, both common adverse effects of treatment with prostaglandin $E_{1}$. Judicious use of inotropic support is initiated if evidence of low cardiac output is detected. Infusion of dopamine at a rate of 3 to $5 \mu \mathrm{g} / \mathrm{kg}$ per minute usually results in improved 
ventricular function. High-dose inotropic support should be used with caution because it can result in increased SVR (systemic vascular resistance) and cause a shift in the $\dot{Q}_{\mathrm{p}} / \dot{Q}_{\mathrm{s}}$ ratio to greater than 1. In our institution we do not recommend the use of dopamine as a "standard" since we consider it a "dirty" drug with a lot of potential unexpected effects. Diuretics may be necessary to help alleviate the increased volume load on the right ventricle.

Infants with an unrestrictive inter-atrial communication may be in a stable condition initially, but signs of congestive heart failure may develop as the PVR (pulmonary vascular resistance) decreases. When oxygen saturations are approximately $90 \%$, systemic blood flow may be reduced, resulting in tissue hypoperfusion, metabolic acidosis, and a low cardiac output state. In infants with high oxygen saturation and evidence of tissue hypoperfusion, controlled mechanical ventilation is often initiated to improve the $\dot{Q}_{\mathrm{p}} / \dot{Q}_{\mathrm{S}}$ ratio and systemic cardiac output.

Severe tricuspid regurgitation (TR) could be another issue that complicates the life of the intensivist dealing with a newborn with HLHS. Good preoperative management with mechanical ventilation in order to lower the degree of TR results in a better short-term prognosis of stage 1 Norwood operation. (4) The goal of respiratory management is to increase pulmonary vascular resistance and decrease systemic vascular resistance. The $\dot{Q}_{\mathrm{p}} / \dot{Q}_{\mathrm{S}}$ ratio can be manipulated by increasing PVR by increasing the $\mathrm{PaCO}_{2} \cdot \mathrm{PaCO}_{2}$ can be increased by adding supplemental inspired carbon dioxide, a potent pulmonary vasoconstrictor, to the ventilator circuit. This approach for increasing $\mathrm{PaCO}_{2}$ is preferred over hypoventilati- on, which may lead to atelectasis. PVR can also be increased by decreasing the concentration of inspired oxygen by adding supplemental nitrogen gas to attain a fraction of inspired oxygen of 0.17 to 0.19 . PVR can also be increased by maintaining the hematocrit at greater than 0.40 , a state that optimizes oxygen-carrying capacity and increases the viscosity of the blood. Although these medical management strategies may provide temporary palliation, infants with marked pulmonary overcirculation and systemic hypoperfusion benefit from early surgical correction, because the methods to reverse this situation have limited effectiveness. Infants with HLHS who are born with a severely restricted or no inter-atrial communication, a rare occurrence, have profound hypoxemia. In fact, morbidity and mortality remain high in the subset of patients with an intact or very restrictive atrial septum. (10) The severe restriction of blood flow across the atrial septum results in a life-threatening situation and these patients, which present with severe cyanosis and hemodynamic instability, require urgent postnatal cardiac catheterization to relieve the septal obstruction and improve oxygenation. (11) Relief of the obstruction can be achieved by a balloon atrial septostomy or blade septostomy at the time of cardiac catheterization or a surgical atrial septectomy. The tenuous condition of these infants makes each of these interventions high risk. The choice of intervention depends on the severity of the obstruction, the infant's cardiac anatomy and physiology, and the experience of the available medical and surgical team.

\section{Keep the brain in mind}

Special attention should be paid to the prevention of brain injury to avoid poor neurodevelopmental outcome. Children with HLHS have an increased prevalence of central nervous system (CNS) abnormalities, which traditionally have been attributed to chronic cyanosis and multiple exposures to cardiopulmonary bypass. (12) However, there is a theory, supported by identification of white matter injury in term neonates with HLHS before surgery, that the impaired antegrade aortic blood flow and consequent reduced cerebral blood flow adversely impact the CNS development in such babies. (13) Unfortunately it seems to have a great impact on the overall success of treatment for children with HLHS, in terms of a definitive satisfactory outcome. Presurgical brain injury is common even among children with the best prognostic characteristics who receive care in tertiary centers. Thus, the majority of surviving children who are born with hypoplastic left heart will not have normal psychomotor development at age one year and parents should be counseled accordingly. (14)

\section{Conclusion}

Care for infants with HLHS is complex and often multiple specialists are involved. Certainly, the best way to manage a newborn with critical heart disease is to diagnose the defect prenatally in order to arrange delivery near experienced pediatric cardiac surgery institutions. Nevertheless, despite an increase in the number of newborns with complex congenital heart disease and a growing percentage of patients with single-ventricle physiology, it is possible to care for this particular group of patients and achieve an acceptable mortality risk, even in centers with no pediatric cardiac surgery facilities, if good preoperative management protocols are followed. (15) 


\section{REFERENCES}

1. Hall RJ, Nelson WP, Blake HA, Geiger JP. Massive Pulmonary Arteriovenous Fistula in the Newborn. Circulation 1965;31:762-7.

2. Izukawa T, Mullholland HC, Rowe RD, Cook DH, Bloom KR, Trusler GA, et al. Structural heart disease in the newborn. Arch Disease Childhood 1979;54:281-5

3. Ravinaskar C, Tabbutt S, Wernovsky G. Critical care in cardiovascular medicine. Curr Opin Pediatr 2003;15:443-53.

4. Baba K, Ohtsuki S, Kamada M, Kataoka K, Ohno N, Okamoto Y, et al. Preoperative management for tricuspid regurgitation in hypoplastic left heart syndrome. Ped Internat 2009;51:399-404.

5. Fricker FJ. Hypoplastic Left Heart Syndrome - Diagnosis and early management. NeoReviews 2008;9:253.

6. Hoffmann GM, Ghanayem NS, Kampine JM, Berger S, Mussatto KA, Litwin SB, et al. Venous saturation and the anaerobic threshold in neonates after the Norwood procedure for hypoplastic left heart syndrome. Ann Thorac Surg 2000;70:1515-21.

7. Singh GH, Fong LV, Salmon AP, Keeton BR. Study of low dosage prostaglandin - usages and complication. Eur Heart J 1994;15:377-81.

8. Lewis AB, Freed MD, Heymann MA, Roehl SL, Kensey RC. Side effects of therapy with prostaglandin E1 in infants with critical congenital heart disease. Circulation 1981;64: 893-8.

9. Thiebaud B, Michelakis E, Wu XC, Harry G, Hashimoto K, Archer SL. Sildenafil Reverses O2 constriction of the rabbit Ductus Arteriosus by inhibiting Type 5 phosphodisesterase and activating BK Ca Channels. Ped Res 2002;52:19-24.

10. Rychik J, Rome JJ, Collins MH, DeCampli WM, Spray TL. The hypoplastic left heart syndrome with intact atrial septum: atrial morphology, pulmonary vascular histopathology and outcome. J Am Coll Cardiol 1999;34:554-60.

11. Atz AM, Feinstein JA, Jonas RA. Preoperative management of pulmonary venous hypertension in hypoplastic left heart syndrome with restrictive atrial septal defect. Am J Cardiol 1999;83:1224-8.

12. Newburger JW, Jonas RA, Wernovsky G. A comparison of the preoperative neurologic effects of hypothermic circulatory arrest versus low-flow cardiopulmonary bypass in infant heart surgery. N Engl J Med 1993;329:1057-64.

13. Miller SP, McQuillen PS, Hamrick S, Xu D, Glidden DV, Charlton N. Abnormal brain development in newborns with congenital heart disease. N Engl J Med. 2007;357:1928-38.

14. Tabbutt S, Nord AS, Jarvik GP. Neurodevelopmental outcomes after staged palliation for hypoplastic left heart syndrome. Pediatrics 2008;121:476-83.

15. Dorfman AT, Marino BS, Wernovsky G, Tabbutt S, Ravishankar C, Godinez RI, et al. Critical heart disease in the neonate: Presentation and outcome at a tertiary care center. Pediatr Crit Care Med 2008;9:193-202. 